\title{
A modified shape context method for shape based object retrieval
}

\author{
Radhika Mani Madireddy ${ }^{1}$, Pardha Saradhi Varma Gottumukkala², Potukuchi Dakshina Murthy ${ }^{3}$ and \\ Satyanarayana Chittipothula ${ }^{4^{*}}$
}

\begin{abstract}
The complexity in shape context method and its simplification is addressed. A novel, but simple approach to design shape context method including Fourier Transform for the object recognition is described. Relevance of shape context, an important descriptor for the recognition process is detailed. Inclusion of information regarding all the contour points (with respect to a reference point) in computing the distribution is discussed. Role of similarity checking the procedure details regarding the computation of matching errors through the alignment transform are discussed. Present case of shape context (for each point with respect to the centroid) descriptor is testified for its invariance to translation, rotation and scaling operations. Euclidean distance is used during the similarity matching. Modified shape context based descriptor is experimented over three standard databases. The results evidence the relative efficiency of the modified shape context based descriptor than that reported for other descriptor of concurrent interests.
\end{abstract}

Keywords: Object recognition; Shape representation; Feature extraction; Distance measure

\section{Introduction}

Although significant progress is witnessed in the field of automated object recognition, it is still remains challenging task (Zhang and Lu 2004; Iyer et al. 2005) from the broad purview of machine learning and computer vision processes of contemporary requirements. The shape of an object contains (Forsyth and Mundy 1999) an important, unique and characteristic features of the object. The shape based methods consider either the contour or the entire region of the object. The consideration of contour involves less representative points in comparison with the region based methods (Nixon and Aguado 2002). The region-based methods consider the global information (all the pixels within a shape) for the design of the descriptor which involves the geometrical moments (Hu 1962; Flusser 2000), Zernike moments (ZM) (Teague 1980; Khotanzad 1990), pseudo- Zernike moments (Belkasim et al. 1991), Legendre moments (Teague 1980), and Tchebichef moments (Mukundan et al. 2001), generic Fourier descriptor (FD) (Zhang and Lu 2002),

\footnotetext{
* Correspondence: chsatyanarayana@yahoo.com

${ }^{4}$ Department of CSE, Jawaharlal Nehru Technological University Kakinada, Kakinada, AP 533003, India

Full list of author information is available at the end of the article
}

compounded image descriptor ( $\mathrm{Li}$ and Lee 2005), shape matrix (Goshtasby 1985), the grid technique (Lu and Sajjanhar 1999) and shock graph (Sebastian et al. 2004; Siddiqi et al. 1999) etc. However, the contour based representation is reported to be more efficient (Yang et al. 2008). Several recently reported contour based methods rely on viz., Fourier transform (Zahn and Roskies 1972; Wallace and Wintz 1980; Kunttu et al. 2006), curvature scale space (CSS) (Mokhtarian and Mackworth 1986; Abbasi et al. 1999, 2000), wavelet transform (Chauang and Kuo 1996; Yadav et al. 2007), contour displacement (Adamek and O'Connor 2004), chain codes (Junding and Xiaosheng 2006), autoregressive (Dubois and Glanz 1986), Delaunay triangulation (Tao and Wi 1999), multiresolution polygonal (Day et al. 2004) robust symbolic representation (Daliri and Torre 2008), distance sets (Grigorescu and Petkov 2003), elastic matching (Attalla and Siy 2005) etc techniques for the design of the shape descriptor. Basing on the consideration of shape boundaries (Petrakis et al. 2002; Arica and Vural 2003; Bartolini et al. 2005; Lateckia et al. 2005; Alajlan et al. 2007), dynamic programming (DP) technique is also adopted to achieve high accuracy rate. The DP based techniques suffer from being computationally expensive and get reduced

\section{实}


to be impractical for large databases, despite the fact that they offer better performance.

Generally, the descriptor relevant to the shape context (Belongie et al. 2002) method for object recognition is developed with an established correspondence between the point sets. The procedure combines the shape context information with the information formatted by using thin plate spline (Bookstein 1989) processing. Due to the proven simplicity and capability of discrimination, the shape context based methods proficiently proposed in the literature (Dubois and Glanz 1986; Tao and Wi 1999; Day et al. 2004; Daliri and Torre 2008; Grigorescu and Petkov 2003; Attalla and Siy 2005; Petrakis et al. 2002; Arica and Vural 2003; Bartolini et al. 2005; Lateckia et al. 2005; Alajlan et al. 2007; Belongie et al. 2002; Bookstein 1989; Mori and Malik 2003; Thayananthan et al. 2003; Zhang and Malik 2003; Salve and Jondhale 2010). Recently, Xin Shu proposed Contour Points Distribution Histogram (CPDH) (Shu and Xiao Jun 2011) for the shape context method. The shape matching process which speaks out the performance of a descriptor is dealt in different ways. The Zucker et al (Siddiqi et al. 1999) has developed shock graph grammar and the relevant tree matching algorithm. The spectral distance (based on diffusion geometry, heat trace) estimated through the Laplacian transform is also used for matching (Bronstein and Kokkinos 2010; Bronstein and Bronstein 2011; Konukoglu et al. 2013). On the other hand, the Fourier transform based matching procedures are is also popular (Cem Direko glu and Nixon 2011; Xingyuan and Zongyu 2013; Ghazal et al. 2009; Ghazal et al. 2012).

In the wake of the results reported in the area of shape context based object recognition techniques involving a wide variety of design of description and matching measures, it serves that the utility of the Fourier based descriptors for the shape context based recognition presents a superior method rather than the contour based methods. Hence, the authors propose for the design of a novel hybrid contour based shape descriptor which is constructed with respect to the centroid, while the feature vector is estimated by a 1D Fourier transform. The shape toning phase is involves the Euclidean Distance to enhance the quality.

The paper is organized in three sections. Introduction to the computerized object recognition method is presented in section-Introduction. Methodology adopted for the present shape context technique is presented in sectionMethodology along with the information for indices to evaluate its performance. The results obtained by adopting present method to the standard databases and their trends are presented in section-Results and discussion along with the relevant discussions of performance.

\section{Methodology}

A multi staged novel and hybrid shape context based scheme for the object recognition process is proposed.
The phase wise information during the processing is presented in section-Design of system, while the proposed indices to estimate the performance are presented in section-Performance.

\section{Design of system}

The details of various stages involved with the proposed object recognition by using shape context are schematically depicted in Figure 1. The proposed system consists of four successive steps viz:

(i) Shape representation with contour

(ii) Computation of Shape Context

(iii) Construction of histogram for each bin of shape context

(iv) Shape description by using Fourier Transform

The descriptor is further expected to a training stage viz., shape toning and ranking. An overview of all these stages of processing implies that the shape based object recognition system includes the salient features of stages, such as shape representation, shape description and shape toning. Contour based shape representation is considered as the initial step of processing. The second step includes description of the shape representation points. Belongie Shape Context (BSC) (Belongie et al. 2002) is popularly used method for describing the shape of the object. During the second step, the contour of the given object is described (Figure 2(a)) by constructing the BSC. During the construction of BSC, the angle between any two points (on log polar transform) is measured with respect to constant center on $\mathrm{x}$ axis given by the Equation-(1).

$$
\theta(\mathrm{x}, \mathrm{y})=\tan ^{-1}\left(\frac{\mathrm{y}_{2}}{\mathrm{y}_{1}}\right)
$$

where:

$\theta(x, y)$ is the angle measurement between two points $x$ and $y$,

$\mathrm{y}_{2}$ is the $y$ coordinate of the first point,

$y_{1}$ is the $y$ coordinate of the second point.

To test the invariance property of the BSC, a Modified Shape Context (MSC) is presently proposed (Figure 2(b)). The MSC measures the angle between any two points with respect to the centroid. If the total no. of contour points are $\mathrm{Z}$ then the farthest two points will be selected and the angle between these points is measured by the Equation-(2).

$$
\theta(\mathrm{x}, \mathrm{y})=\tan ^{-1}\left(\frac{\mathrm{m}_{2}-\mathrm{m}_{1}}{1+\mathrm{m}_{2}+\mathrm{m}_{1}}\right)
$$

where: 


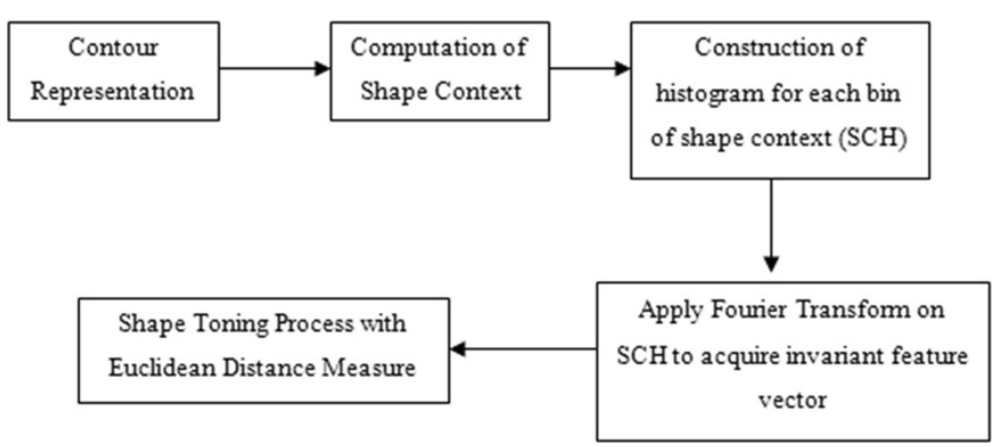

Figure 1 Schematic diagram of the proposed system with shape context.

$\theta(x, y)$ is the angle measurement of a point $(x, y)$, $\mathrm{m}_{1}$ is the slope of the line between first point and second point,

$\mathrm{m}_{2}$ is the slope of the line between first point and center point.

A histogram containing each bin of the shape context $(\mathrm{SCH})$ is constructed for each part of the shape context to enable the shape context to viable to various transformations. In the wake of the fact that the Fourier Transform is widely used transformations (Ghazal et al. 2009; Zhang and $\mathrm{Lu}$ 2005) for object recognition problems and its coefficients are found to be invariant to symmetry operations (i.e. translation, rotation and scaling etc). The size of the shape representation points is an important and influential factor that optimizes the utility of Fourier transformation. Hence, in the shape signature generation process, the sampling is considered as a mandatory step. Some of the sampling methods like Equal Point (EP), Equal Angle (EA) and Equal Arc Length (EAL) (Zhang and Lu 2003) are considered presently. EAL is expected to yield for a better equal space (Peter and Otterloo 1991) than the other two methods.
By using EAL, the representation of the contour is restricted to $\mathrm{N}$-number of points. The proposed method uses EAL method to sample the finite number of contour points. For a given contour signal, the 1-D Fourier transform is given as;

$$
\mathrm{FD}_{\mathrm{n}}=\frac{1}{\mathrm{~N}} \sum_{\mathrm{t}=0}^{\mathrm{N}-1} \mathrm{~s}(\mathrm{t}) \times \exp \left(\frac{-\mathrm{j} 2 \pi \mathrm{nt}}{\mathrm{N}}\right)
$$

where:

$\mathrm{s}(\mathrm{t})$ represents the 1-D contour signal,

$\mathrm{N}$ represents number of representative points of the contour, $\mathrm{n}=0,1,2, \ldots, \mathrm{N}-1$ and,

$\mathrm{FD}_{\mathrm{n}}$ represents $\mathrm{n}^{\text {th }}$ Fourier descriptor.

Using Equation (3), the required Fourier Descriptors of size ' $N$ ' are generated. Further, the extracted features are testified for their invariance to translation, rotation and scaling operations (performed over the set of images). In the wake of the fact that the proposed descriptor is obtained with respect to the centroid, the obtained

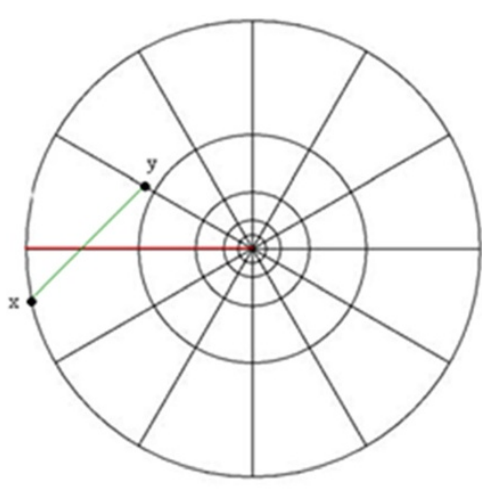

(a1)

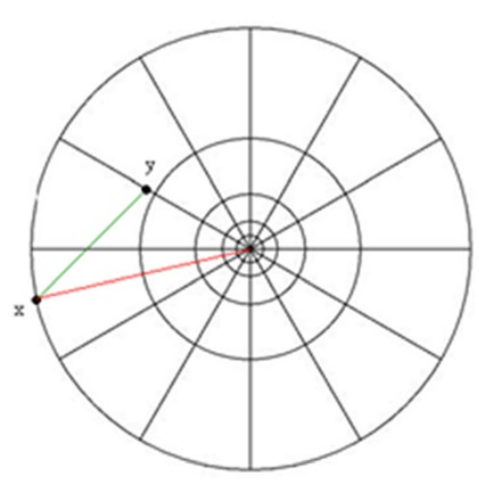

(b1)

Figure 2 Example of constructing (a) Belongie Shape Context (b) Modified Shape Context signature for a contour point wrt the corresponding farthest point. 


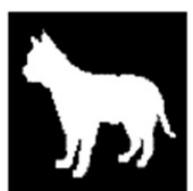

(a1)

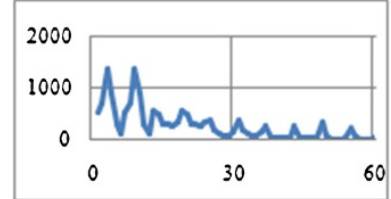

(a2)

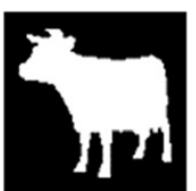

(b1)

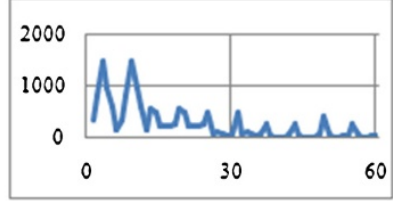

(b2)

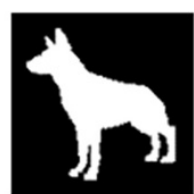

(c1)

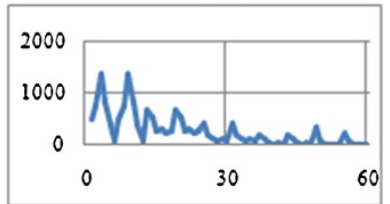

(c2)

Figure 3 Proposed descriptor for three Animal group Images (a1) Animal 3 (a2) MSC signature of Animal 3 (b1) Animal 5 (b2) MSC signature of Animal 5 (c1) Animal 7 (c2) MSC signature of Animal 7.

features are expected to be invariant to translation. The possible finite (and stipulated) magnitude of the values for the features vouches for the rotation invariance. For the present method, the scaling invariance is also presented by involving the process of dividing the features with the first feature value. In the third step, the feature vector is constructed, which describes the entire shape features of the object.

To further improve the quality of proposed methods, the global information of the object is also considered. For this, experiments are conducted with considering different global descriptors and identified that three global descriptors (GD) are efficient to represent the global shape information. The GD feature vector, viz., $\{\mathrm{S}, \mathrm{C}, \mathrm{A}\}$ contains the measures of solidity, circularity and aspect ratio is computed for the given object.

In the fourth step, the shape toning process is executed. In the shape toning process, the distance measures (Ghazal et al. 2009, 2012) used is viz., the Euclidean distance (ED). The distance measure between two objects shape context vectors is given by the Equation (4). In this, the average global distance of global feature vectors is directly added to the Euclidean distance of the Fourier descriptor feature vector.

The distance between two shape context vectors including the object global feature vectors is given by the Equation (5).

$$
\mathrm{D}(\mathrm{TE}, \mathrm{TR})=\mathrm{ED}(\mathrm{TE}, \mathrm{TR})+\frac{\mathrm{D}_{\mathrm{X}}(\mathrm{TE}, \mathrm{TR})}{3}
$$

where:

ED (TE, TR) represents the Euclidean Distance between the test and trained shapes and, $\mathrm{D}_{\mathrm{X}}$ (TE,TR) represents the Global distance between the test and trained shapes.

$$
\mathrm{D}_{\mathrm{X}}(\mathrm{TE}, \mathrm{TR})=\sum_{\mathrm{X}} \frac{\left|\mathrm{X}^{\mathrm{TE}}-\mathrm{X}^{\mathrm{TR}}\right|}{\max \left(\mathrm{X}^{\mathrm{TR}}\right)}
$$

where:

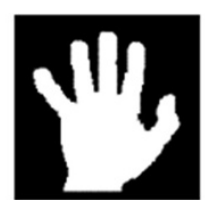

(a1)

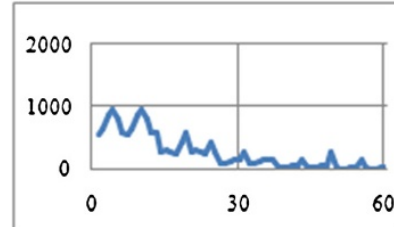

(a2)

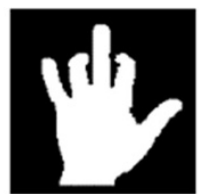

(b1)

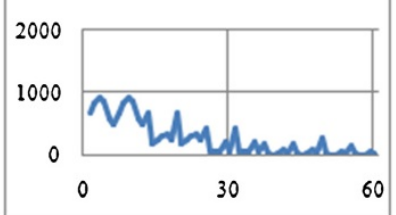

(b2)

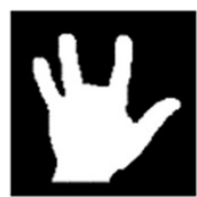

(c1)

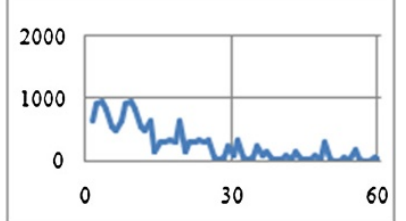

(c2)

Figure 4 Proposed descriptor for three Hand group Images (a1) Hand 8 (a2) MSC signature of Hand 8 (b1) Hand 9 (b2) MSC signature of Hand 9 (c1) Hand 11 (c2) MSC signature of Hand 11. 


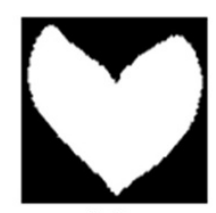

(a1)

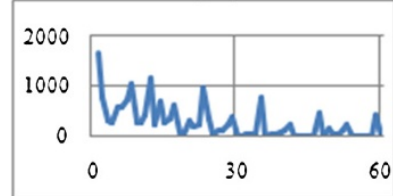

(a2)

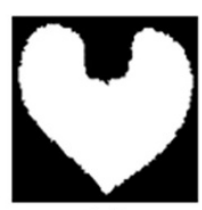

(b1)

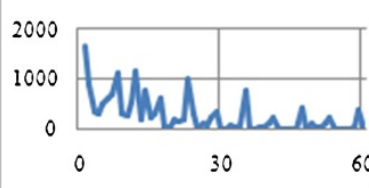

(b2)

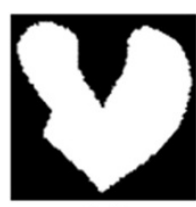

(c1)

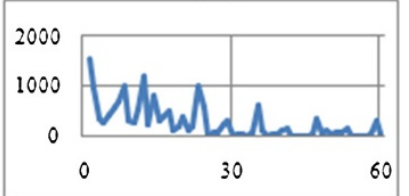

(c2)

Figure 5 Proposed descriptor for three Heart group Images (a1) Heart 7 (a2) MSC signature of Heart 7 (b1) Heart 11 (b2) MSC signature of Heart 11 (c1) Heart 12 (c2) MSC signature of heart 12.

$X$ represents the $G D$ vector $\{S, C, A R\}$,

$\mathrm{X}^{\mathrm{TE}}$ represents the GD feature of the test shape and,

$\mathrm{X}^{\mathrm{TR}}$ represents the GD feature of the trained shape.

According to the specificity of the data of distance measurement, the distances are further rearranged in ascending order and are assigned with ranks. However, the system is also enabled to recognize and register the top ranked images.

The standard databases (Sikora 2001; Sebastian et al. 2001) used for the evaluation of shape descriptors presently are Kimia $\{$ K-99, K-216\} and MPEG CE-1 Set $B$. It is noticed that the Set B database with 70 groups and each group with 20 images. It characteristically includes rotated, scaled, skewed and defected shapes. However the K-99 database which consists of 9 groups, each group with 11 images. It is known to include the partially occluded shapes. The K-216 database with 18 groups, each group with 12 images, it represents a sub database of Set B, and contains partially occluded shapes.

\section{Performance}

The performance of various object recognition schemes reported (Shu and Xiao Jun 2011; Ghazal et al. 2009, 2012) so far employ different measures. Among them, precision and recall are considered as important measures, while they verbally quantify the similarity measurement. Precision (P) and Recall (R) are defined by:

$$
\mathrm{P}=\frac{\mathrm{x}}{\mathrm{y}}
$$

where:

$x$ denotes the true recognition results, $y$ denotes the total recognized result and, $\mathrm{P}$ denotes the precision.

$$
\mathrm{R}=\frac{\mathrm{x}}{\text { groupsize }}
$$

where:

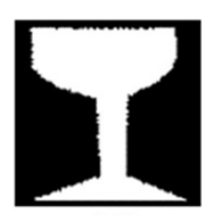

(a1)

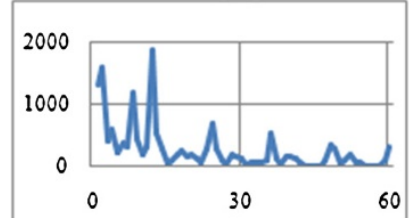

(a2)

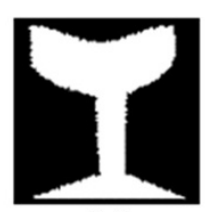

(b1)

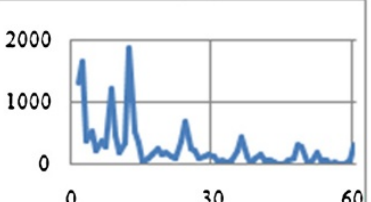

(b2)

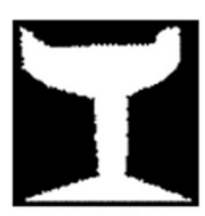

(c1)

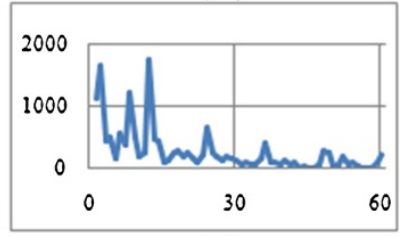

(c2)

Figure 6 Proposed descriptor for three Fly group Images (a1) Fly 1 (a2) MSC signature of Fly 1 (b1) Fly 4 (b2) MSC signature of Fly 4 (c1) Fly 10 (c2) MSC signature of Fly 10. 


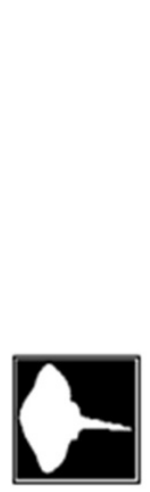

(a)

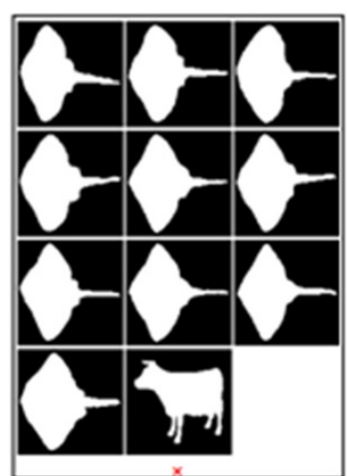

(b)

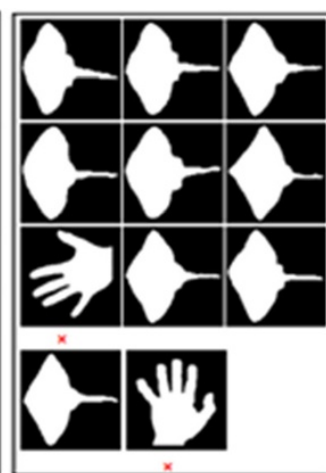

(c)

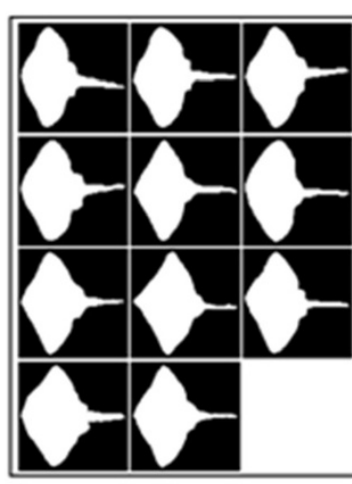

(d)

Figure 7 Retrieval results of Key2 test image from K-99 database (a) Original image (b) ZMD result (c) BSC+GD result (d) MSC+GD result.

$\mathrm{R}$ denotes the Recall,

$\mathrm{x}$ denotes the true recognition results and,

group size denotes the maximum true recognition result.

The Average Precision value for each recall is computed. This value is affirmatively grouped as two categories viz., Low Recall (LR), High Recall (HR). The Average Precision for Low Recall (APLR) denotes the average precision for recalls less than or equal to 50. In contrast, the Average Precision for High Recall (APHR) represents the average precision for recalls greater than 50. The False Detection Rate (FDR) for each of the image is also estimated by:

$$
\mathrm{FDR}=\frac{\mathrm{z}}{\mathrm{y}}
$$

where:
FDR denotes the False Detection Rate, $\mathrm{z}$ denotes the false recognition result and, $y$ denote the total recognized result.

The average FDR (AFDR) value of all test images corresponding to each database is estimated. Apart from the usual recognition rate, the Average Processing Time (APT) is also estimated for each query in the shape toning stage. The proposed descriptor is compared with 4 standard descriptors viz., Angular Radial Transform Descriptor (ARTD) (Zhang et al. 2008), Moment Invariant Descriptor (MID) (Zhang et al. 2008), Zernike Moment Descriptor (ZMD) (Tiagrajah and Razeen 2011) and Curvature-Scale-Space-Descriptor (CSSD) (Tiagrajah and Razeen 2011). A specific feature size of 35 for ARTD $(\mathrm{n}<3, \mathrm{~m}<12), 6$ for MID, 34 for ZMD (order from 2 to 10) is adopted. The CSSD feature size is varying from that for one image to another image since number of peaks is varying. All the cited metrics viz.,

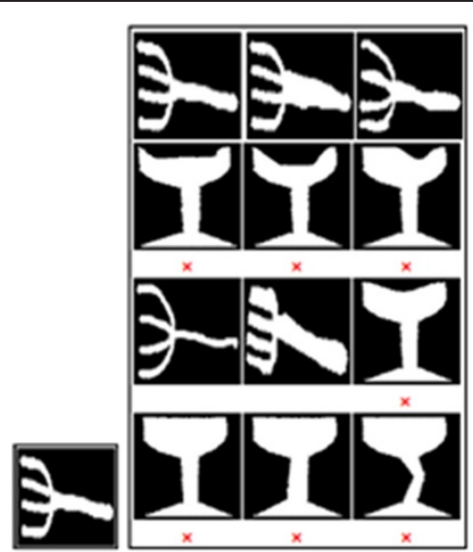

(a)

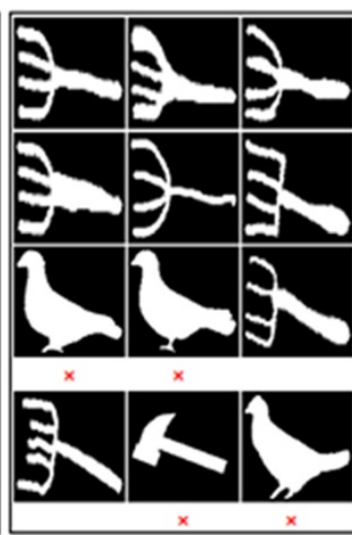

(c)

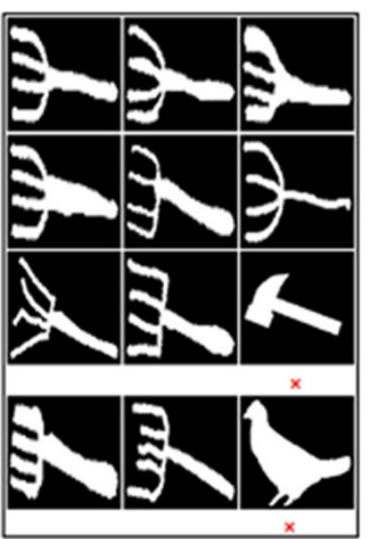

(d)

Figure 8 Retrieval results of Fork12 test image from K-216 database (a) Original image (b) ZMD result (c) BSC+GD result (d) MSC+GD result. 


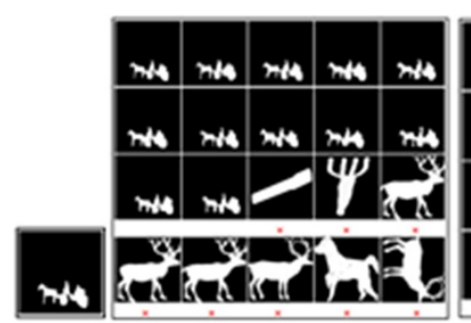

(a) (b)

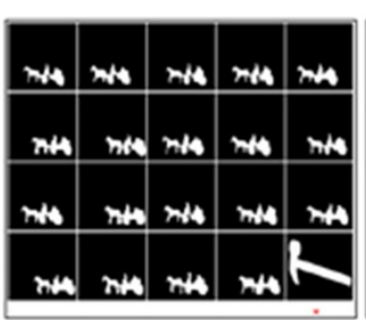

(c)

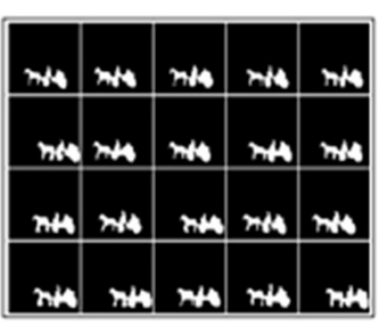

(d)

Figure 9 Retrieval results of Carriage16 test Image from Set B database (a) Original image (b) ZMD result (c) BSC+GD result (d) MSC+GD result.

APLR, APHR, AFDR and APT are evaluated to estimate the performance for the proposed descriptor (with inclusion of GD), ARTD, MID, ZMD and CSSD.

\section{Results and discussion}

Shape context based object recognition is estimated as detailed in section-Design of system for the input of standard databases. The trends of the results that follow various approaches are presented in the following sub section-Processing of modified shape context based object recognition. The relative performance of the proposed descriptor is also analyzed in the sectionPerformance evaluation in the wake of the other reported methods.

\section{Processing of modified shape context based object recognition}

The shape context is constructed with 60 bins. Then for each contour point, the angle is measured (i.e. BSC and $\mathrm{MSC}$ ) within the range of one full rotation i.e. from $0^{\circ}$ to $360^{\circ}$. A histogram is generated that corresponds to each and every bin of shape context. The histograms, thus constructed are presented in Figures 3, 4, 5, and 6 corresponding to four different image groups (i.e. animal, hand, heart and glass) as accessed from set B, K-99 and

Table 1 The APLR and APHR values for various descriptors with Set B database

\begin{tabular}{lccc}
\hline & \multicolumn{3}{c}{ Avg. precision } \\
\cline { 2 - 4 } Descriptor & Recall < $\mathbf{5 0} \%$ & Recall $>\mathbf{5 0} \%$ & Average \\
\hline BSC & 82.65 & 49.37 & 66.01 \\
MSC & 84.02 & 49.56 & 66.79 \\
BSC + GD & 84.48 & 52.85 & 68.67 \\
MSC + GD & 88.83 & 55.58 & 72.21 \\
ARTD & 82.10 & 45.69 & 63.90 \\
MI & 79.54 & 44.50 & 62.02 \\
ZMD & 82.56 & 45.62 & 64.09 \\
CSSD & 78.61 & 41.81 & 60.21 \\
\hline
\end{tabular}

K-216 databases. Figures 3(a1), -3(b1) and -3(c1) contains three original images of animal group (animal-3, animal-5 and animal-7); the Figures 4(a1), -(b1) and -(c1) pertain to the three original images of hand group (hand8, hand9, hand11); the Figures 5(a1), -(b1) and -(c1) give three original images of heart group (heart-7, heart-11 and heart-12); and Figures 6(a1), -(b1) and -(c1) contain three original images of fly group (Fly1, Fly4 and Fly10). The corresponding Modified shape context (MSC) histogram is illustrated in Figures 3(a2), 4, 5, 6(a2), Figures 3(b2), 4, 5, 6(b2) and Figures 3(c2), 4, 5, 6(c2) respectively. It is clearly noticed from Figures 3, 4, 5, 6, that the MSC histogram is found to be similar for the different shapes within the same group; while it exhibits difference between those of one group compared to the other. Basing on 1-D shape signal, the Fourier descriptors (FD) are generated. The experiments are conducted with varying size of feature vector. From this, it is identified that the first ten features of the FD are consistent. Hence, they are used to design the feature vector.

In the present object recognition process, Euclidean Distance (ED) measure of performance is estimated between the target and test objects, while they are allocated with ranking according to their distance. In accord with the established procedures, the top n-ranked

Table 2 The APLR and APHR values for various descriptors with K-99 database

\begin{tabular}{lccc}
\hline & \multicolumn{3}{c}{ Avg. precision } \\
\cline { 2 - 4 } Descriptor & Recall $<=\mathbf{5 0} \%$ & Recall $>\mathbf{5 0} \%$ & Average \\
\hline BSC & 86.01 & 51.25 & 68.63 \\
MSC & 89.11 & 56.87 & 72.99 \\
BSC + GD & 87.21 & 57.82 & 72.52 \\
MSC + GD & 90.77 & 62.43 & 76.60 \\
ARTD & 84.26 & 45.72 & 64.99 \\
MI & 81.96 & 44.74 & 63.35 \\
ZMD & 89.61 & 61.37 & 75.49 \\
CSSD & 82.32 & 44.11 & 63.22 \\
\hline
\end{tabular}


Table 3 The APLR and APHR values for various descriptors with K-216 database

\begin{tabular}{lccc}
\hline & \multicolumn{3}{c}{ Avg. precision } \\
\cline { 2 - 4 } Descriptor & Recall $<=\mathbf{5 0 \%}$ & Recall $>\mathbf{5 0 \%}$ & Average \\
\hline BSC & 88.50 & 57.59 & 73.05 \\
MSC & 90.16 & 61.18 & 75.67 \\
BSC + GD & 91.01 & 64.00 & 77.50 \\
MSC + GD & 91.50 & 66.00 & 78.75 \\
ARTD & 81.35 & 44.67 & 63.01 \\
MI & 80.14 & 46.04 & 63.09 \\
ZMD & 88.94 & 61.71 & 75.33 \\
CSSD & 80.12 & 44.97 & 62.55 \\
\hline
\end{tabular}

objects are used to estimate the Precision and Recall parameters. The ' $n$ ' notifies the group size for $\{$ Set B:20, K99:11 and K-216:12\} sets. For each database, the accuracy for the retrieval results corresponding to top 'n' (group size of the corresponding database) number of images is estimated and illustrated in Figures 7, 8, 9 respectively. For K-99 database:- The top 11 ranked images correspond to the query image Key2 (as estimated by using ZMD, BSC + GD and MSC + GD), while they are presented in the Figures 7(a)-(d). The Figure 7(a) corresponds to the Key2 query image and Figure 7(b) corresponds to the retrieval results with ZMD descriptor. Figure 7 (c) gives the retrieval results for BSC + GD descriptor; and Figure 7(d) vouches for the retrieval results with MSC + GD descriptor. For K-216 database:- The top 12 ranked images corresponding to the query image Fork12 are estimated by using ZMD, BSC + GD and MSC + GD as presented in Figures 8(a)-(d). The Figure 8(a) illustrates the Fork12 query image and Figure 8(b) speaks for the retrieval results with ZMD descriptor.
Figure $8(\mathrm{c})$ gives the retrieval results with $\mathrm{BSC}+\mathrm{GD}$ descriptor, and Figure $8(\mathrm{~d})$ gives the retrieval results with $\mathrm{MSC}+\mathrm{GD}$ descriptor.

For Set B database:- the top 20 ranked images corresponds to the query image of Carriage 16 by using ZMD, $\mathrm{BSC}+\mathrm{GD}$ and MSC + GD as presented in the Figures 9 (a)-(d). The Figure 9(a) gives the Carriage16 query image, Figure 9(b) gives the retrieval results with ZMD descriptor, Figure 9(c) gives the retrieval results with $\mathrm{BSC}+\mathrm{GD}$ descriptor and Figure 9(d) gives the retrieval results with $\mathrm{MSC}+\mathrm{GD}$ descriptor. Overview of cited Figures 7(a), 8, 9(d) suggests that, the MSC + GD descriptor performs better for retrieval of more relevant images with relatively strong correspondence than that with the other descriptors.

\section{Performance evaluation}

The yield of APLR and APHR values for the descriptor with the currently proposed distance measure is analyzed. In the wake of the four other standard descriptors, the aspect of compatibility (with three databases) is also explored, while the results are presented in Tables 1, 2, 3 respectively. From these results, it is clearly evident that the presently proposed descriptor out performs the other descriptors regarding all the three standard databases. However, among the presently considered descriptors, the CSSD descriptor is found to accompany with a lower performance, followed by that of MID, ARTD. However, the case of ZMD resulted for the next higher performance. But, for Set B database, the ZMD is yielding the highest result. From Table 1, it is found that the proposed MSC + GD happen to be influential to increase the APLR value of ZMD. It is also found to significantly increase the APHR value of ARTD. For K-99 and K-216 databases,

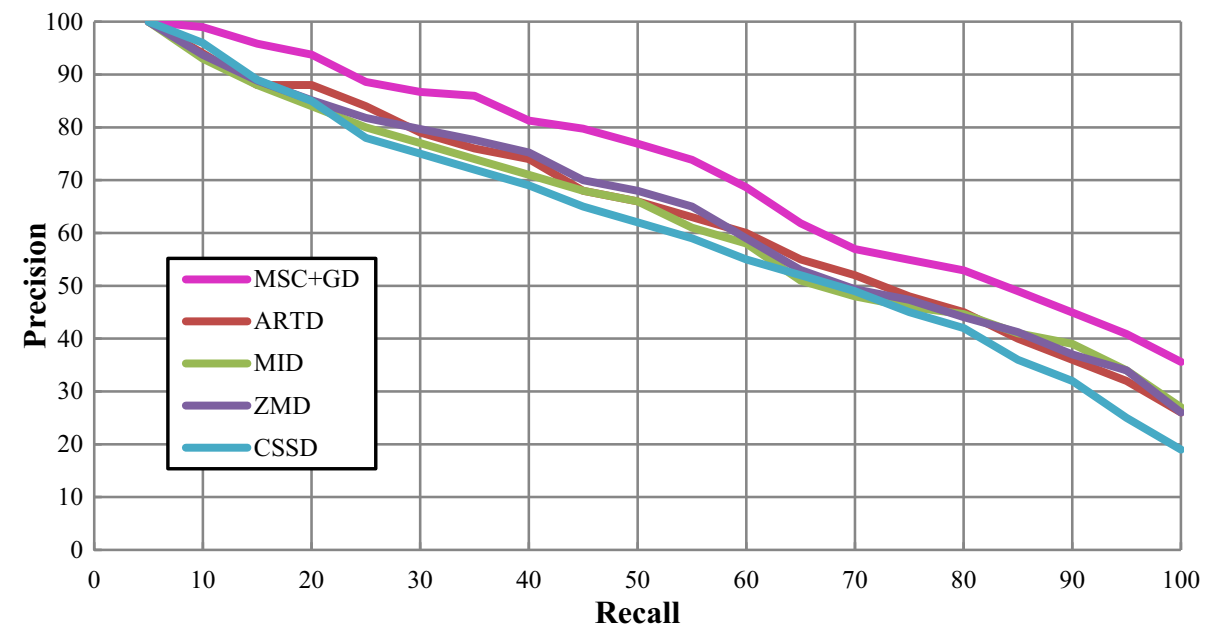

Figure 10 The PR graph for various descriptors with set B database. 


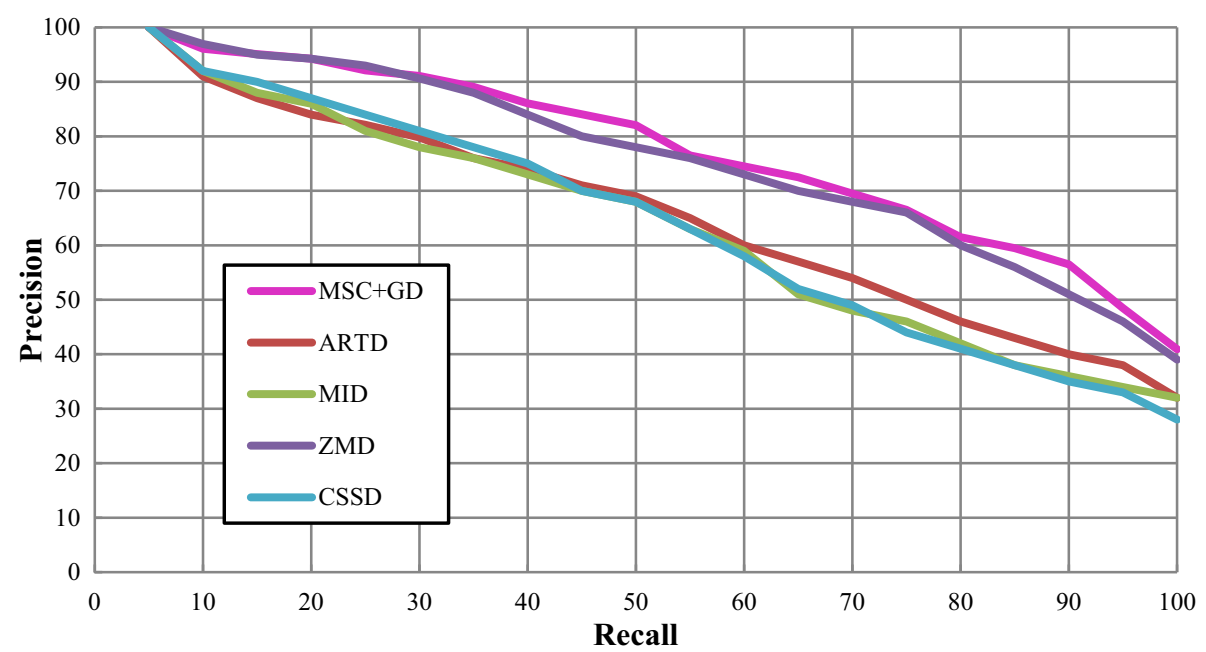

Figure 11 The PR graph for various descriptors with K-99 database.

the ZMD is giving distinctly improved APLR and APHR values than with the other descriptors. From the Tables 2 and 3; it is evident that the proposed MSC + GD is accompanied with an improved performance in terms of enhancement of APLR and APHR.

The PR plots for these five descriptors comparing to the set B, K-99 and K-216 are presented in Figures 10, 11 and 12 respectively. Figure 10 reveals that all the five descriptors are yielding considerable enhanced performance with regard to the precision measure for the Set B database in the range of low recalls. At higher recalls, the proposed MSC + GD measure is found to result for improved precision measure, rather than the other standard descriptors. The proposed CSD is found to increase the precision measure marginally at lower recalls i.e. $\leq 50$, bit, it is found to significantly increase the precision at higher recalls i.e. $>50$. The PR plot for K-99 database is depicted in Figure 11. From this figure, it is observed that the ZMD outperforms other standard descriptors with increased precision measure in the range of both lower and higher recalls. An overview of the results infers that the proposed MSC + GD measure is considerable increase in precision measure at lower recalls ranged between 40 and 50 and higher recalls ranged between 60 to 70 and 90 to 100 . The precision measure is found to attain considerable improvement at higher recalls i.e. at 80 to 100 . Figure 12 describes the PR plot of various descriptors for K-216 database. The ZMD in this PR plot is found to be superior, rather than other standard descriptors at lower and higher recalls. Thus the proposed MSC + GD measure is giving increased precision measure regarding PR plots at both lower and higher recalls.

Other Performance measures viz., Average False Discovery Rate (AFDR) and Average Processing Time (APT) are

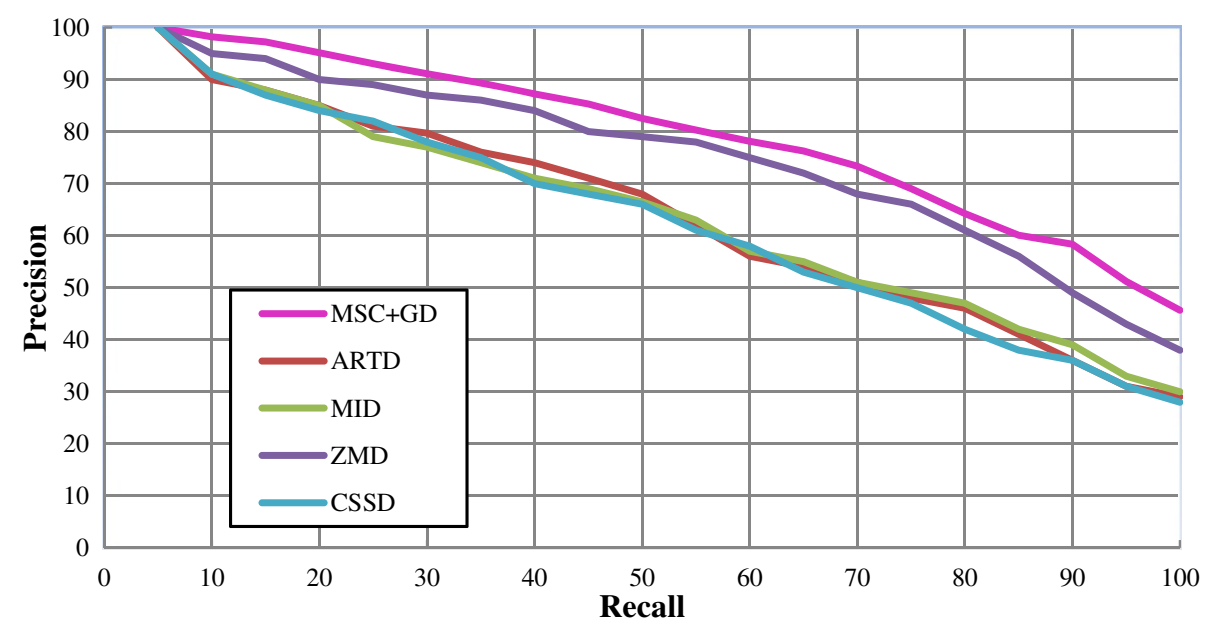

Figure 12 The PR graph for various descriptors with K-216 database. 
Table 4 The AFDR for various descriptors with three databases

\begin{tabular}{lccc}
\hline Descriptor & \multicolumn{3}{c}{ AFDR } \\
\cline { 2 - 4 } & Set B & K-99 & K-216 \\
\hline MSC + GD & 0.70 & 0.72 & 0.73 \\
ARTD & 0.90 & 0.85 & 0.86 \\
MID & 0.91 & 0.81 & 0.84 \\
ZMD & 0.78 & 0.76 & 0.76 \\
CSSD & 0.84 & 0.88 & 0.89 \\
\hline
\end{tabular}

also estimated as detailed in section-2.2, while the estimated AFDR values for the three databases are presented in Table 4. It is observed that the proposed MSC + GD results for a lower value for all the three databases. Since, the APT measure is argued to exhibit profound influence on shape toning stage (for each of the shape descriptor), it is also estimated for all the three databases, and presented in Table 5. The proposed MSC + GD is found to yield for less APT value in comparison with other descriptors. Therefore, basing the observed trends of performance measures, it is argues that the proposed descriptor exhibits higher efficiency. As a measure of performance, the retrieval rate with bull's eye score (Cem Direko glu and Nixon 2011) is also estimated. This measure involves the calculation of the ratio of the total number of shapes (i.e. from the same class) to the highest possible number of shapes in the same database. The estimated bull's eye score for top 40 results in Set B database is presented in Table 6 . It is clear that Inner Distance Shape Context (IDSC) is yielding highest score when compared with the others. However, as this includes complicated dynamic programming procedure, the simple Euclidean distance measure for the proposed descriptor is argued to be more efficient retrieval parameter.

\section{Conclusions}

- Shape context based description is proved to be efficient when compared with various other standard descriptors with respect to various performance measures viz., APLR, APHR, AFDR and APT.

Table 5 APT of various descriptors with set B database

\begin{tabular}{lc}
\hline Descriptor & APT \\
\hline MSC + GD & 0.0011 \\
ARTD & 0.0017 \\
MID & 0.0302 \\
ZMD & 0.0017 \\
CSSD & 2.1640 \\
\hline
\end{tabular}

Table 6 Bull's eye score for various descriptors with set B database

\begin{tabular}{ll}
\hline Descriptor & Score \% \\
\hline CSS (Mokhtarian et al. 1997) & 75.44 \\
Visual Parts (Latecki and Rolf 2000) & 76.45 \\
SC + TPS (Belongie et al. 2002) & 76.51 \\
Aligning Curves (Sebastian et al. 2003) & 78.16 \\
SSC (Xie et al. 2008) & 79.92 \\
CPDH + EMD (Cem Direko glu and Nixon 2011) & 76.56 \\
General Model (Tu and Yuille 2004) & 80.03 \\
MSC + GD & 81.64 \\
IDSC + DP (Ling \& Jacobs 2007) & 85.40 \\
\hline
\end{tabular}

- The proposed descriptor improves the precision measures at high recalls when compared with the low recalls thus enabling more relevant objects to be recognized.

- With less feature vector size, the proposed descriptor enables the object recognition system to be efficient with less APT and AFDR measures.

\section{Competing interests}

PSVG areas of interest include Object Oriented Technologies, Information Retrieval, Algorithms, Computer Networks, and Image Processing. PDM areas of interests are Experimental soft condensed matter, Liquid crystalsdesign and characterization, Theory of spectroscopy, chemistry - design of supermolecules, Face Recognition, Image Processing, and optical character recognition. SCH area of interest includes Image processing, Database Management Systems, Speech Recognition, Pattern recognition and network security.

\section{Authors' contributions}

RMM carried out the shape context studies, participated in the development of the proposed shape descriptor and drafted the manuscript. PSVG participated in the design of the study with the shape toning process, and drafted the manuscript. PDM participated in the design of the proposed shape descriptor with Fourier Transformation, and drafted the manuscript. SCH participated in the design of the study with feature extraction, and drafted the manuscript. All authors read and approved the final manuscript.

\section{Author details}

'Department of CSE, Pragati Engineering College, Surampalem, AP 533437, India. ${ }^{2}$ Department of IT, SRKR Engineering College, Bhimavaram, AP 534204, India. ${ }^{3}$ Department of Physics, Jawaharlal Nehru Technological University Kakinada, Kakinada, AP 533003, India. ${ }^{4}$ Department of CSE, Jawaharlal Nehru Technological University Kakinada, Kakinada, AP 533003, India.

Received: 10 September 2014 Accepted: 31 October 2014 Published: 15 November 2014

\section{References}

Abbasi S, Mokhtarian F, Kittler J (1999) Curvature scale space image in shape similarity retrieval. Multimed Syst 7:467-476

Abbasi S, Mokhtarian F, Kittler J (2000) Enhancing CSS-based shape retrieval for objects with shallow concavities. Image Vis Comput 18:199-211

Adamek T, O'Connor NE (2004) A multi scale representation method for non rigid shapes with a single closed contour. IEEE Trans Circuits Syst Video Technol 14:742-753

Alajlan N, Rube IE, Kamel MS, Freeman G (2007) Shape retrieval using triangle-area representation and dynamic space warping. Pattern Recogn 40:1911-1920 
Arica N, Vural FTY (2003) BAS: a perceptual shade descriptor based on the beam angle statistics. Pattern Recogn Lett 24:1627-1639

Attalla E, Siy P (2005) Robust shape similarity retrieval based on contour segmentation polygonal multi resolution and elastic matching. Pattern Recogn 38:2229-2241

Bartolini I, Ciaccia P, Patella M (2005) WARP: accurate retrieval of shapes using phase of Fourier descriptors and time warping distance. IEEE Trans Pattern Anal Mach Intell 27:142-147

Belkasim SO, Shridhar M, Ahmadi M (1991) Pattern recognition with moment invariants: a comparative study and new results. Pattern Recogn 24:1117-1138

Belongie S, Malik J, Puzicha J (2002) Shape matching and object recognition using shape contexts. IEEE Trans Pattern Anal Mach Intell 24(4):509-522

Bookstein FL (1989) Principal warps: thin-plate-splines and decomposition of deformations. IEEE Trans Pattern Anal Mach Intell 11(6):567-585

Bronstein MM, Bronstein AM (2011) Shape recognition with spectral distances. IEEE Trans Pattern Anal Mach Intell 33(5):1065-1071

Bronstein MM, Kokkinos I (2010) Scale invariant heat kernel signatures for non rigid shape recognition. IEEE Conf Comput Vision Pattern Recogn (CVPR) 1704-1711, doi:10.1109/CVPR.2010.5539838

Cem Direko glu, Nixon MS (2011) Shape classification via image-based multi scale description. Pattern Recogn 44:2134-2146

Chauang G, Kuo C (1996) Wavelet descriptor of planar curves: theory and applications. IEEE Trans Image Process 5:56-70

Daliri MR, Torre V (2008) Robust symbolic representation for shape recognition and retrieval. Pattern Recogn 41:1782-1798

Day AM, Arnold DB, Havemann S, Fellner DW (2004) Combining polygonal and subdivision surface approaches to modeling and rendering of urban environments. Comput Graph 28(4):497-507

Dubois SR, Glanz FH (1986) An autoregressive model approach to two- dimensional shape classification. IEEE Trans Pattern Anal Mach Intell 8:55-65

Flusser J (2000) On the independence of rotation moment invariants. Pattern Recogn 33:1405-1410

Forsyth D, Mundy J (1999) Shape, contour and grouping in computer vision. Lect Notes Comput Sci 1681:1-3

Ghazal AE, Basir O, Belkasim S (2009) Farthest point distance: a new shape signature for Fourier descriptors. Signal Process Image Comm 24:572-586

Ghazal AE, Basir O, Belkasim S (2012) Invariant curvature-based Fourier shape descriptors. J Vis Commun Image R 23:622-633

Goshtasby A (1985) Description and discrimination of planar shapes using shape matrices. IEEE Trans Pattern Anal Mach Intell 7:738-743

Grigorescu C, Petkov N (2003) Distance sets for shape filters and shape recognition. IEEE Trans Image Process 12(10):1274-1286

Hu M (1962) Visual pattern recognition by moment invariants. IRE Trans Inform Theor IT 8:115-147

Iyer N, Jayanti S, Lou K, Kalyanaraman Y, Ramani K (2005) Three dimensional shape searching: state of the art review and future trends. Comput Aided Des 37:509-530

Junding S, Xiaosheng W (2006) Chain Code Distribution-Based Image Retrieval. International Conference on Intelligent Information Hiding and Multimedia Signal Processing, China, pp 139-142

Khotanzad A (1990) Invariant image recognition by Zernike moments. IEEE Trans Pattern Anal Mach Intell 12:489-497

Konukoglu E, Glocker B, Criminisi A, Pohl KM (2013) WESD-weighted spectral distance for measuring shape dissimilarity. IEEE Trans Pattern Anal Mach Intell 35(9):2284-2297

Kunttu I, Lepisto L, Rauhamaa J, Visa A (2006) Multi scale fourier descriptors for defect image retrieval. Pattern Recogn Lett 27:123-132

Latecki LJ, Lakamper R (2000) Shape similarity measure based on correspondence of visual parts. IEEE Trans Pattern Anal Mach Intell 22(10):1185-1190

Lateckia $\sqcup$, Lakaempera R, Wolter D (2005) Optimal partial shape similarity. Image Vis Comput 23:227-236

Li S, Lee MC (2005) Effective invariant features for shape-based image retrieval. J Am Soc Inf Sci Technol 56:729-740

Ling $H$, Jacobs DW (2007) Shape classification using the inner distance. IEEE Trans Pattern Anal Mach Intell 29(2):286-299

Lu G, Sajjanhar A (1999) Region-based shape representation and similarity measure suitable for content based image retrieval. Multimed Syst $7: 165-174$
Mokhtarian F, Mackworth A (1986) Scale-based description and recognition of planar curves and two-dimensional shapes. IEEE Trans Pattern Anal Mach Intell 8:34-43

Mokhtarian F, Abbasi F, Kittler J, Smeulders AWM, Jain R (1997) Efficient and robust retrieval by shape content through curvature scale space. In: Image Databases and Multi-Media Search. World Scientific Publishing, Singapore, pp 51-58

Mori G, Malik J (2003) Recognizing objects in adversarial clutter: breaking a visual CAPTCHA. IEEE Conf Comput Vision Pattern Recogn 1:1063-6919

Mukundan R, Ong SH, Lee PA (2001) Image analysis by Tchebichef moments. IEEE Trans Image Process 10:1357-1364

Nixon MS, Aguado AS (2002) Feature Extraction and Image Processing, 1st edn. Newnes Publishers, Burlington MA, pp 247-287

Peter J, Otterloo V (1991) A contour- Oriented Approach to Shape Analysis. Prentice Hall, Hertfordshire UK

Petrakis EGM, Diplaros A, Milios E (2002) Matching and retrieval of distorted and occluded shapes using dynamic programming. IEEE Trans Pattern Anal Mach Intell 24:1501-1516

Salve SG, Jondhale KC (2010) Shape matching and object recognition using shape contexts. In: Computer Science and Information Technology (ICCSIT), vol 9. 3rd IEEE International Conference, pp 471-474

Sebastian TB, Klein PN, Kimia BB (2001) Recognition of shapes by editing shock graphs. ICCV 1:755-762

Sebastian T, Klein P, Kimia B (2003) On aligning curves. IEEE Trans Pattern Anal Mach Intell 25(1):116-125

Sebastian TB, Klein PN, Kimia BB (2004) Recognition of shapes by editing their shock graphs. IEEE Trans Pattern Anal Mach Intell 26(5):550-571

Shu X, Xiao Jun W (2011) A novel contour descriptor for 2D shape matching and its application to image retrieval. Image Vis Comput 29:286-294

Siddiqi K, Shokoufandeh A, Dickinson SJ, Zucker SW (1999) Shock graphs and shape matching. Int J Comput Vis 35(1):13-32

Sikora T (2001) The MPEG-7 visual standard for content description- an overview. IEEE Trans Circuits Syst Video Technol 11(6):696-702

Tao Y, Wi G (1999) Delaunay Triangulation for Image Object Indexing: A Novel Method for Shape Representation. Seventh SPIE Symposium on Storage and Retrieval for Image and Video Databases San Jose, CA, pp 631-642

Teague M (1980) Image analysis via the general theory of moments. J Opt Soc Am 70:920-930

Thayananthan A, Stenger B, Torr PHS, Cipolla R (2003) Shape context and chamfer matching in cluttered scenes. IEEE Conf Comput Vision Pattern Recogn 1:127-133

Tiagrajah VJ, Razeen AASM (2011) An enhanced shape descriptor based on radial distances. IEEE Int Conf Signal Image Process Appl (ICSIPA) 472-477, doi:10.1109/ICSIPA.2011.6144073

Tu Z, Yuille A (2004) Shape matching and recognition-using generative models and informative features. Proc Eur Conf Comput Vis 3:195-209

Wallace TP, Wintz PA (1980) An efficient three dimensional aircraft recognition algorithm using normalized Fourier descriptors. Comput Graph Image Process 13:99-126

Xie J, Heng P, Shah M (2008) Shape matching and modeling using skeletal context. Pattern Recogn 41(5):1756-1767

Xingyuan W, Zongyu W (2013) A novel method for image retrieval based on structure elements' descriptor. J Vis Commun Image R 24:63-74

Yadav RB, Nishchal NK, Gupta AK, Rastogi VK (2007) Retrieval and classification of shape-based objects using Fourier, generic Fourier, and waveletFourier descriptors technique: a comparative study. Opt Lasers Eng 45:695-708

Yang M, Kpalma K, Ronsin J (2008) A Survey of shape feature extraction techniques. In: Pen Y (ed) Pattern Recognition., pp 43-90

Zahn T, Roskies RZ (1972) Fourier descriptors for plane closed curves. IEEE Trans Comput 21:269-281

Zhang D, Lu G (2002) Shape-based image retrieval using generic Fourier descriptor. Signal Process Image Commun 17:825-848

Zhang D, Lu G (2003) A comparative study of curvature scale space and Fourier descriptors for shape based image retrieval. J Vis Commun Image Represents 14(1):39-57

Zhang D, Lu G (2004) Review of shape representation and description techniques. Pattern Recogn 37(1):1-19

Zhang D, Lu G (2005) Study and evaluation of different fourier methods for image retrieval. Image Vis Comput 23(11):33-49 
Zhang H, Malik J (2003) Learning a discriminative classifier using shape context distances. IEEE Conf on Comput Visi Pattern Recogn 1:242-247

Zhang G, Ma ZM, Tong Q, He Y, Zhao T (2008) Shape Feature Extraction using Fourier Descriptors with Brightness in Content-Based Medical Image Retrieval. International Conference on Intelligent Information Hiding and Multimedia Signal Processing, pp 71-74

doi:10.1186/2193-1801-3-674

Cite this article as: Madireddy et al:: A modified shape context method for shape based object retrieval. SpringerPlus 2014 3:674.

Submit your manuscript to a SpringerOpen ${ }^{\circ}$ journal and benefit from:

- Convenient online submission

- Rigorous peer review

- Immediate publication on acceptance

- Open access: articles freely available online

- High visibility within the field

- Retaining the copyright to your article

Submit your next manuscript at $\gg$ springeropen.com 